\title{
Biblioterapia. Recomendaciones terapéuticas de libro en la autoterapia ampliada
}

\author{
Bibliotherapy. Therapeutic book recommendations in \\ extended self-therapy
}

\section{Biblioterapia. Recomendações de livros terapêuticos em autoterapia estendida}

\author{
Analía Verónica Losada ${ }^{1}$ ORCID: 0000-0003-0488-4651 \\ Antonio Martín Román² ORCID: 0000-0002-5207-3901
}

\footnotetext{
${ }^{1}$ Universidad de Flores. Facultad de Psicología y Ciencias Sociales. Ciudad Autónoma de Buenos Aires, Argentina. Correo electrónico: analia_losada@yahoo.com.ar.

${ }^{2}$ Universidad de Flores. Psicología con Orientación en Metodología de la Revisión. Facultad de Psicología y Ciencias Sociales. Ciudad Autónoma de Buenos Aires, Argentina. Correo electrónico: cdu340@gmail.com.
}

\section{Resumen}

La recomendación de libros como instrumento terapéutico ha sido el eje central de la presente investigación, que postula a la biblioterapia como la terapia y tratamiento a través de los libros. Con el objetivo de conocer las encomiendas terapéuticas de libros en términos de autoterapia ampliada, se realizaron encuestas a 81 profesionales de la psicología de 8 países. 77 participantes son de América (57 de Argentina, 14 de Colombia, 3 de Brasil, 2 de Perú y 1 de Ecuador); 1 de Europa (España), y 3 de África (2 de Angola y 1 de Mozambique). Entre los participantes, 73 - que representan el 90,12\%- explicitaron que se valen de la biblioterapia como recurso. Los terapeutas expusieron sobre los autores y obras que sugieren a sus pacientes. Entre los motivos de recomendación, el de mayor frecuencia ha sido el de continuar aspectos trabajados durante la sesión a modo de autoterapia ampliada. Como conclusión se destaca la concordancia con los autores en los resultados aportados por los partícipes y se sugiere el uso de la biblioterapia en diferentes dispositivos de tratamiento.

Palabras clave: BIBLIOTERAPIA; TERAPIA; PSICOLOGÍA CLÍNICA; PSICOLOGÍA SOCIAL; AUTOTERAPIA. 


\begin{abstract}
Book recommendations as a therapeutic instrument have been this investigation's central axis, positing Bibliotherapy as treatment through books. With the goal of determining which books were being recommended as extensions to therapy, 81 psychological professionals from 8 countries were surveyed. 57 of those 81 were from Argentina, 14 from Colombia, 3 from Brazil, 2 from Peru and 1 from Ecuador; with a total of 77 South Americans. Additionally, 3 African participants took part: 2 from Angola and 1 from Mozambique. Finally, there was 1 based in Spain. Among those surveyed, 73 (90.12\%) made explicit that they use Bibliotherapy as a resource. Therapists presented authors and works they suggest to their patients. Among the reasons for recommendation, the most frequent has been to expand on subjects covered during the session. In conclusion, there is a significant consensus in favor of Bibliotherapy and its use in different treatment devices is recommended.
\end{abstract}

Keywords: BIBLIOTHERAPY; THERAPY; CLINICAL PSYCHOLOGY; SOCIAL PSYCHOLOGY; SELF-THERAPY.

\title{
Resumo
}

A recomendação do livro como instrumento terapêutico tem sido o eixo central desta pesquisa, que postula a biblioterapia como terapia e tratamento por meio do livro. Para conhecer os pedidos de livros terapêuticos em termos de autoterapia estendida, foram realizadas pesquisas com 81 profissionais de psicologia de 8 países. 77 participantes são da América (57 da Argentina, 14 da Colômbia, 3 do Brasil, 2 do Peru e 1 do Equador); 1 da Europa (Espanha) e 3 da África (2 de Angola e 1 de Moçambique). Entre os participantes, 73 - o que representa 90,12\% - afirmaram utilizar a biblioterapia como recurso. Os terapeutas falaram sobre os autores e as obras que sugerem aos seus pacientes. Dentre os motivos de recomendação, o mais frequente tem sido a continuidade dos aspectos trabalhados durante a sessão como uma autoterapia prolongada. Em conclusão, destaca-se a concordância com os autores nos resultados fornecidos pelos participantes e sugere-se o uso da biblioterapia em diferentes dispositivos de tratamento.

Palavras-chave: BIBLIOTECA DE TERAPIA; TERAPIA; PSICOLOGIA CLINICA; PSICOLOGIA SOCIAL; AUTOTERAPIA.

Fecha de recibido: $31 / 05 / 2021$

Fecha de aceptado: 07/09/2021 


\section{Introducción}

La biblioterapia se constituye como la terapia y el tratamiento a través de los libros, es decir, la utilización de textos como recursos terapéuticos, considerados herramientas válidas para los procesos de crecimiento y recuperación en el marco de la psicoterapia (Losada, 2020; Román, 2019; Román, 2020.)

A la vez, abordar el concepto del sentido de la vida y su importancia dentro de la psicología lleva a recorrer una vasta literatura interdisciplinaria. El sentido de la vida se ha afrontado como un fin que puede trascender las situaciones límites de la existencia. A este respecto, existe el término neurosis noógena, que fue sugerido por Frankl (2013) para explicar una sintomatología particular, no necesariamente depresiva, psicogenética, con accesos de angustia o de ansiedad, con un cierto grado de conflicto intrapsíquico reconocible o consciente, que puede presentar alguna expresión psicosomática concomitante. Las emociones más características son la desesperanza, la ausencia de un propósito de vida, la falta de valores prosociales. La neurosis noógena se cursa conjuntamente con una apatía notoria y un profundo sentimiento de desamparo. No se trata de una enfermedad, pero es importante notar que puede, eventualmente, emerger ante situaciones de fuerza mayor, tales como la pandemia social provocada por el coronavirus, e implicar una serie de cambios en la vida cotidiana, por ejemplo, un mayor aislamiento. Según Frankl (2012), se trata de una neurosis existencial frente al vacío del sentido de la vida, en la que subyacen, con mayor o menor nivel de notoriedad, aspectos depresivos y tanáticos. Frente a una neurosis noógena resulta de suma importancia, de acuerdo con la literatura sobre el tema, afrontar los cambios producidos por factores exógenos y desarrollar habilidades logoterapéuticas, tal como puede ser la biblioterapia, es decir, la posibilidad de encontrar un goce estético en la lectura amena de un libro. La eventualidad de disfrutar un buen libro trasciende el hecho concreto del padecimiento, para resaltar aspectos de la vida, real o simbólica, que hacen posible focalizar la atención del lector adviniendo en su auxilio. 
La literatura permite una nueva construcción de la realidad, ya que estimula el pensamiento crítico y la acción reflexiva. También involucra el análisis de la propia realidad del lector y de sus experiencias de vida.

Como refiere Clemente (1964), el libro es un instrumento de excitación de experiencias psíquicas correspondientes a todas las particularidades de la mentalidad en la que estas experiencias se producen, y la lectura contiene valores que al proyectarse en nosotros pueden hacen resurgir sentimientos estéticos que aguardan su oportunidad liberadora. De allí que la experiencia de leer otorgue la sensación de libertad y el habitar otros mundos diferentes a los de uno, hecho que enriquece la propia vida con nuevas situaciones que muchas veces no se tendría la posibilidad de vivir.

La duración de las sesiones de psicoterapia, en promedio de una hora y de frecuencia semanal, conllevan que el profesional realice indicaciones que el paciente pueda proseguir durante la semana y dar cuenta de lo trabajado en la siguiente consulta. Se denomina «autoterapia» porque la realiza el paciente sin la presencia del psicólogo y «ampliada» puesto que se desarrolla fuera del espacio de la sesión, aunque la indicación de la realización se acuerda en el marco de la entrevista. Entre las recomendaciones de autoterapia resultan clásicas y eficaces pedirle al paciente que vea determina película o que lea un libro especifico. La autoterapia ampliada da cuenta de fenómenos a desplegar como tarea entre una sesión y la siguiente, buscando continuar el trabajo que desarrolló durante la sesión, ampliando y aumentando los beneficios terapéuticos (Losada, 2021).

\subsection{Planteo del problema}

La biblioterapia es uno de los recursos terapéuticos más accesibles en la era digital. Existen muchos estudios que fundamentan su valor como instrumento moderno de intervención clínica. Además, su uso y aplicación se extiende a lo largo de la historia de la humanidad. No obstante, la difusión de este instrumento de intervención entre los psicólogos contemporáneos parece no corresponder con los antecedentes y los basamentos teóricos propios de este recurso. Al mismo tiempo, se encuentran pocos trabajos que den cuenta de las dificultades de la tarea biblioterapéutica propiamente dicha. Este hecho evidencia más aún la falta de 
promoción respecto de su aplicación cotidiana, por lo que resulta de valor indagar cuál es el grado de familiaridad que existe con la biblioterapia entre los profesionales de la salud.

Lograr un acercamiento desde la biblioterapia al ámbito de la clínica facilita descubrir el universo de libros que pueden favorecer, mediante una intervención psicodiagnóstica desde la clínica, no solo cuál es la mirada profesional y actual que se tiene de este instrumento, sino la validación de cierta literatura por encima de otra a través de un criterio exclusivamente terapéutico de los libros. En este sentido, una indagación sobre el uso biblioterapéutico de los libros en la clínica actual es también un antecedente que permitirá facilitar la tarea a futuros profesionales. Por ello, en principio sería interesante indagar con qué frecuencia se encuentra comúnmente aplicada la técnica biblioterapéutica en la clínica psicológica contemporánea. Importa asimismo investigar cuáles son los criterios que adopta un psicoterapeuta frente a un paciente cuando recomienda la lectura de un determinado libro o autor, con lo cual se permitirá aportar a la biblioterapia nuevo material de valor para todos los futuros profesionales de la salud o expertos, por su proximidad con las prácticas del rol profesional.

\subsection{Antecedentes}

En relación con la lectura y sus implicaciones terapéuticas, es posible mencionar algunos trabajos documentados recientemente en los que varios autores proponen puntualmente la perspectiva de la biblioterapia.

Bardají (2016), en su artículo Cómo afrontar la pérdida con Proust: un apunte de biblioterapia, sugiere que la comprensión del ser humano basado en lo observable del comportamiento es solo un enfoque posible de la psicología contemporánea, existen otros que se interesan en otros registros, como la sabiduría.

La lectura en sus implicaciones en la instrumentación clínica es actualmente utilizada de diversas maneras. Kroeff y Reverté (2007) han creado un dispositivo a partir del cual indagan ciertas frases tomadas de los libros para abordar situaciones de duelo en grupos psicoterapéuticos conformados por personas que han quedado viudas recientemente. 
La experiencia de creación de bibliotecas para pacientes da cuenta de su aplicabilidad en los centros de salud. Deberti Martins (2011) postula esta implementación ponderando la lectura como fenómeno complejo de construcción de sentido y de reconstrucción de sí mismo, primordialmente en situaciones de vulnerabilidad psíquica y física, como es el caso de las personas hospitalizadas.

Román (2019) en su tesis Imaginarios sociales de exreclusos sobre la lectura y el sentido de la vida presenta, a su vez, otros episodios recogidos en su investigación respecto del sentido de la vida relacionado con la lectura.

Del Río (2011) trabaja su propio dispositivo de lectura destinado a grupos de tercera edad, para favorecer el contacto con el sentido de la vida; la vejez es una etapa en la que el hombre continúa desarrollándose, sigue vivo el deseo de encontrarle sentido a su vida, de encontrar el para qué de su vida.

\subsection{Fundamentos teóricos}

En su genealogía contemporánea, el libro y la biblioterapia pueden ser ubicados en su dimensión clínica desde que Frankl dio el discurso Das Buch als Therapeutikum (El libro como terapia) en la Feria del Libro de Austria en Viena en 1975. Este versaba sobre la posibilidad de la sanación por medio de la lectura. En la ocasión, mencionó casos en los cuales un libro, un texto, un relato habían salvado la vida de personas, las habían alejado de ideas suicidas, les habían permitido entender la necesidad de construir una vida con sentido.

Así, por lo anteriormente expuesto, para Frankl la actividad de la lectura queda completamente asociada a la búsqueda de sentido y, por eso, puede desprenderse de ella que posee un gran valor terapéutico.

Por lo tanto, la biblioterapia en su acepción moderna cobra mayor importancia a partir de los desarrollos de Viktor Frankl y la logoterapia, que le otorgan un valor terapéutico vinculado al libro o a cualquier estructura lingüística capaz de proveer un sentido a la persona en contacto con él. A partir de algunos autores contemporáneos, se ha encontrado que la biblioterapia puede aplicarse en forma individual o grupal, inclusive puede estar destinada a poblaciones que padecen un tipo de neurosis - la neurosis noógena - cuya característica principal es una 
ausencia del sentido de la vida; tal como puede ser comprobado en diversas comunidades vulnerables como son los internos en instituciones totales, en cárceles o en asilos para ancianos, los refugiados políticos o los grupos de minorías sociales o étnicas.

\section{Objetivo}

Conocer las recomendaciones terapéuticas de libros en términos de autoterapia ampliada.

\section{Metodología}

Se construyó un cuestionario ad hoc que buscó conocer el uso de la biblioterapia a través de las recomendaciones terapéuticas de lectura de profesionales psicólogos a pacientes en tratamiento. La encuesta diseñada incluyó una serie de preguntas con respuestas de opciones múltiples y también preguntas abiertas, en las que el participante podía explayarse. Las interpelaciones se construyeron sobre la base del conocimiento de los investigadores acerca de la temática, aunando disciplinas como bibliotecología y psicología.

En primer término, y previamente a la realización del cuestionario, cada uno de los psicólogos participantes dio cumplimiento al instrumento del consentimiento informado. Esta herramienta permite que quienes otorgan su contribución puedan retirarse del estudio si así lo sean y regular su participación en el marco de la ética y legislación vigentes (Losada, 2014).

La muestra se conformó con 81 participantes — psicólogos de los continentes América, África y Europa, matriculados en los colegios profesionales conforme la legislación de cada uno de los países — según el siguiente detalle (véase tabla 1):

Tabla 1. Muestra y nacionalidad

\begin{tabular}{|l|c|}
\hline \multicolumn{1}{|c|}{ Nacionalidad } & Cantidad de profesionales \\
\hline Argentina & 57 \\
\hline Colombia & 14 \\
\hline Brasil & 3 \\
\hline Perú & 2 \\
\hline Angola & 2 \\
\hline
\end{tabular}




\begin{tabular}{|l|c|}
\hline Ecuador & 1 \\
\hline España & 1 \\
\hline Mozambique & 1 \\
\hline Total & $\mathrm{n}=81$ \\
\hline
\end{tabular}

Fuente: elaboración propia

\section{Resultados}

A fin de conocer las recomendaciones de libros por parte de profesionales psicólogos a sus pacientes en el marco del tratamiento psicoterapéutico, se les consultó a los copartícipes acerca de si habían sugerido alguna vez un texto a los consultantes. La respuesta fue afirmativa en 73 de los 81 profesionales consultados, que representan el $90,12 \%$ de los casos. A la vez, 8 de los participantes encuestados señalaron que nunca habían sugerido un libro a un paciente, lo que representa el 9,88\%.

En la tabla 2 se presentan los autores seleccionados por los profesionales para la recomendación en el marco del proceso terapéutico, ordenados de modo alfabético. Si bien las sugerencias incluyen autores latinoamericanos y, en especial, de Argentina y Uruguay, las recomendaciones se centran en autores nacidos en los Estados Unidos con textos traducidos al español.

\section{Tabla 2. Autores seleccionados por los profesionales para la recomendación} terapéutica

\begin{tabular}{|l|l|}
\hline Autores & \\
\hline Aberastury, Arminda & Hirigoyen, Marie-France \\
Aguinis, Marcos & Hustvedt, Siri \\
Arendt, Hannah & Huxley, Aldous \\
Auster, Paul & Johnson, Spencer \\
Bacharach, Estanislao & Kertész, Roberto \\
Balaguer, Roberto & Keyes, Marian \\
Barthes, Roland & Kübler-Ross, Elisabeth \\
Bornemann, Elsa & Lukas, Elisabeth \\
Cameron, Anne & Martin-Lugand, Agnès \\
Canoura, Cristina & Méndez, Isabel \\
Carnegie, Dale & Minnicelli, Mercedes \\
Ceberio, Marcelo R. & Miralles, Francesc \\
Chopra, Deepak & Norwood, Robin \\
Cunningham, Michael & Pinkola Estés, Clarissa \\
Davidson, Richard & Riso, Walter \\
De Mello, Anthony & Robbins, Anthony \\
De Saint-Exupéry, Antoine & Ruiz, Miguel Ángel \\
Dolto, Françoise & Sacks, Oliver \\
Dyer, Wayne Walter & Santos, Care \\
\hline
\end{tabular}




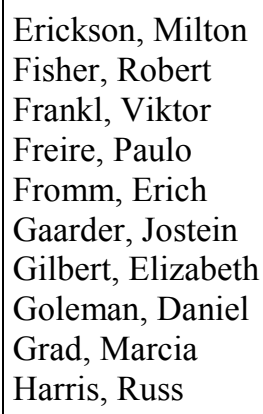

Erickson, Milton

Fisher, Robert

Frankl, Viktor

Freire, Paulo

Fromm, Erich

Gaarder, Jostein

Gilbert, Elizabeth

Goleman, Daniel

Grad, Marcia

Harris, Russ

\author{
Schuzberge, Anne Ancelin \\ Sierra Uribe, Gloria \\ Stamateas, Bernardo \\ Tolle, Eckhart \\ Warren, Rick \\ Weiss, Brian \\ Wilde, Oscar \\ Yalom, Irvin \\ Young, Jeffrey
}

La tabla 3 menciona los títulos de libros recomendados y a sus autores. En el caso de Françoise Dolto, son sugeridas dos obras de su autoría.

Tabla 3. Títulos de libros recomendados y autores respectivos

\begin{tabular}{|c|c|}
\hline Títulos & Autor \\
\hline La adolescencia normal & Arminda Aberastury \\
\hline La novela de mi vida & Marcos Aguinis \\
\hline La banalidad del mal & Hannah Arendt \\
\hline La invención de la soledad & Paul Auster \\
\hline En cambio & Estanislao Bacharach \\
\hline $\begin{array}{l}\text { Hiperconectados: guía para la educación de los nativos } \\
\text { digitales }\end{array}$ & $\begin{array}{l}\text { Roberto Balaguer y Cristina } \\
\text { Canoura }\end{array}$ \\
\hline Fragmento de un discurso amoroso & Roland Barthes \\
\hline Un elefante ocupa mucho espacio & Elsa Bornemann \\
\hline El lugar más bonito del mundo & Anne Cameron \\
\hline Cómo ganar amigos e influir sobre las personas & Dale Carnegie \\
\hline $\begin{array}{l}\text { Cenicientas y patitos feos: de la desvalorización personal } \\
\text { a la buena autoestima }\end{array}$ & Marcelo R. Ceberio \\
\hline Las horas & Michael Cunningham \\
\hline Cuerpos sin edad, mentes sin tiempo & Deepak Chopra \\
\hline El perfil emocional de tu cerebro & Richard Davidson \\
\hline Despierta & Anthony de Mello \\
\hline El principito & Antoine de Saint-Exupéry \\
\hline La causa de los adolescentes & Françoise Dolto \\
\hline Los niños y su derecho a la verdad & Françoise Dolto \\
\hline Tus zonas erróneas & Wayne Dyer \\
\hline Mi voz irá contigo & Milton Erickson \\
\hline Mujeres que corren con los lobos & Clarissa Pinkola Estés \\
\hline El caballero de la armadura oxidada & Robert Fisher \\
\hline La educación como práctica de la libertad & Paulo Freire \\
\hline
\end{tabular}




\begin{tabular}{|c|c|}
\hline El arte de amar & Erich Fromm \\
\hline A pesar de todo decir sí a la vida & Viktor Frankl \\
\hline El hombre en busca de sentido & Viktor Frankl \\
\hline El mundo de Sofia & Jostein Gaarder \\
\hline Come, reza, ama & Elizabeth Gilbert \\
\hline Inteligencia emocional & Daniel Goleman \\
\hline La princesa que creía en los cuentos de hadas & Marcia Grad \\
\hline El acoso moral & Marie-France Hirigoyen \\
\hline Act with love & Russ Harris \\
\hline La mujer temblorosa o la historia de mis nervios & Siri Hustvedt \\
\hline Un mundo feliz & Aldous Huxley \\
\hline ¿Quién se ha llevado mi queso? & Spencer Johnson \\
\hline Análisis transaccional integrado & Roberto Kertész \\
\hline Flores para Algernon & Marian Keyes \\
\hline La morte e el morrer & Elisabeth Kübler Ross \\
\hline En la tristeza pervive el amor & Elisabeth Lukas \\
\hline La gente feliz lee y toma café & Agnès Martin-Lugand \\
\hline Alimentación emocional & Isabel Menéndez \\
\hline Ceremonias mínimas & Mercedes Minnicelli \\
\hline Las mujeres que aman demasiado & Robin Norwood \\
\hline ¿Amar o depender? & Walter Risso \\
\hline Pensar bien, sentirse bien & Walter Riso \\
\hline Despertando al gigante interior & Anthony Robins \\
\hline Los cuatro acuerdos & Miguel Ruiz \\
\hline Alucinaciones & Oliver Sacks \\
\hline El mejor lugar del mundo es aquí mismo & $\begin{array}{l}\text { Care Santos y Francesc } \\
\text { Miralles }\end{array}$ \\
\hline ;Ay, mis ancestros! & Anne Schüzenberger \\
\hline Gente tóxica & Bernardo Stamateas \\
\hline El poder del ahora & Eckhart Tolle \\
\hline Si nos amamos, amémonos bien & Gloria Sierra Uribe \\
\hline
\end{tabular}




\begin{tabular}{|l|l|}
\hline Una vida con propósito & Rick Warren \\
\hline Muchas vidas, muchos maestros & Brian Weiss \\
\hline El retrato de Dorian Gray & Oscar Wilde \\
\hline Mirar al sol & Irvin Yalom \\
\hline Reinventa tu vida & Jeffrey Young \\
\hline
\end{tabular}

Fuente: elaboración propia

Prosiguiendo con el tratamiento de los datos relevados, en la tabla 4 se pondera la frecuencia de la sugerencia terapéutica. El texto de Frankl El hombre en busca de sentido registra 9 recomendaciones, lo que lo constituye como el libro más ofrecido por la muestra de profesionales relevada. A continuación se muestran los resultados relevados, siendo que los textos de Fromm y Fisher El arte de amar y El caballero de la armadura oxidada han sido propuestos en cuatro casos cada uno. En cambio de Bachrach, Inteligencia emocional de Goleman y Tus zonas erróneas de Dyer arrojaron una frecuencia de dos formulaciones y los títulos restantes fueron aludidos en una sola oportunidad cada uno, vease en Tabla 4.

Tabla 4. Título, autor y frecuencia de sugerencia terapéutica

\begin{tabular}{|l|l|c|}
\hline Título & Autor & Frecuencia \\
\hline El hombre en busca de sentido & Viktor Frankl & 9 \\
\hline El caballero de la armadura oxidada & Robert Fisher & 4 \\
\hline El arte de amar & Erich Fromm & 4 \\
\hline En cambio & Estanislao Bachrach & 2 \\
\hline Inteligencia emocional & Daniel Goleman & 2 \\
\hline Tus zonas erróneas & Wayne Dyer & 2 \\
\hline Cada título restante & & 1 \\
\hline
\end{tabular}

Fuente: elaboración propia

Con el fin de conocer los motivos que fundamentan su trabajo en esta línea terapéutica, se preguntó a los entrevistados por qué utilizaban la biblioterapia y recomendaban un texto a sus pacientes. Los profesionales que colaboraron con el presente estudio señalaron en 13 casos que buscaban dar continuidad a lo trabajado en el espacio terapéutico; cifra que representó la mayor motivación. Con relación a estimular la recomendación acerca de la búsqueda del sentido de la vida, surgieron 10 referencias. Respecto del contenido temático y de la similitud de la experiencia personal del entrevistado con cada uno de los motivos que 
llevaron a la indicación de la lectura, se recogieron 8 menciones. Acerca de los intentos de ampliar la perspectiva y aceptación del cambio, así como de fomentar la expresión de las emociones, se citaron como motor de la indicación 6 casos de cada uno. Ofrecer recursos literarios a la hora de elaborar un duelo fue mencionado en 4 de las motivaciones dadas en la presente pesquisa. Las indicaciones para la modificación de creencias rígidas a modo de distorsiones y para ampliar los conocimientos acerca de las disfunciones desde un espacio más ameno (ambas favorecen el cambio y la ampliación del mapa mental) fueron motivadas en tres instancias cada una. El fortalecimiento de vínculos de pareja fue el motivo indicado en dos casos, como así también en dos de los espacios dados, aumentar las elecciones libres. Y, por último, la recomendación a modo de relajación fue el motivo de una de las sugerencias profesionales.

Estos resultados se explicitan a continuación en la tabla 5:

Tabla 5. Motivaciones para la recomendación terapéutica

\begin{tabular}{|l|c|}
\hline \multicolumn{1}{|c|}{ Motivo } & Cantidad \\
\hline $\begin{array}{l}\text { Dar continuidad a un tema puntual trabajado en terapia: } \\
\text { superación de obstáculos, auto esquemas, mitigación de rasgos } \\
\text { ansiógenos o depresivos, dependencias, autoestima }\end{array}$ & 13 \\
\hline Reflexionar sobre cuál es el sentido que se otorga a la vida & 10 \\
\hline $\begin{array}{l}\text { Ofrecer al paciente temáticas que le interesan para ampliar sus } \\
\text { perspectivas y encontrar nuevas }\end{array}$ & 8 \\
\hline Identificar similitud entre la historia, el personaje y el paciente & 8 \\
\hline $\begin{array}{l}\text { Estimular la generación de nuevas formas de comportarse, } \\
\text { facilitar el cambio }\end{array}$ & 7 \\
\hline Facilitar la expresión de emociones. & 7 \\
\hline Reflexionar sobre las relaciones interpersonales & 6 \\
\hline Promover la búsqueda de recursos personales de adaptación & 6 \\
\hline Brindar material de ayuda para la elaboración de duelo & 4 \\
\hline Promover la autoobservación de creencias limitantes o rígidas & 3 \\
\hline $\begin{array}{l}\text { Ampliar conocimientos sobre disfunciones desde un lugar más } \\
\text { ameno }\end{array}$ & 3 \\
\hline Fortalecer el vínculo de pareja & 2 \\
\hline Ampliar la posibilidad de elegir & 1 \\
\hline Facilitar la relajación & \\
\hline
\end{tabular}

Fuente: elaboración propia 
En la tabla 6, se presentan, categorizados por país, los datos acerca de los 8 profesionales que no recomendaron textos. Si bien el número de participantes de Angola y Mozambique no tiene un peso significativo en la muestra, es de destacar que ninguno de los 3 efectuó recomendaciones de textos con fines terapéuticos a sus consultantes.

Tabla 6. Sin recomendaciones de libros por país

\begin{tabular}{|l|c|}
\hline País & Cantidad \\
\hline Argentina & 3 \\
\hline Colombia & 2 \\
\hline Angola & 2 \\
\hline Mozambique & 1 \\
\hline
\end{tabular}

Fuente: elaboración propia

\section{Discusión}

Las contribuciones de Frankl en términos del libro como terapia postulan la posibilidad de la sanación por medio de la lectura, aportando al lector un otorgamiento de sentido y la comprensión de vivencias y situaciones. En línea con estos principios, en la presente investigación se asoció la recomendación de la lectura por parte de los profesionales terapeutas a sus pacientes a las acciones tendientes a mitigar ansiedades así como a reflexionar acerca del sentido y de la búsqueda del sentido.

La utilización de la biblioterapia como recurso terapéutico se reconoce en el presente estudio como un instrumento de implementación frecuente, asequible y de aceptación por parte de los psicólogos. Este desarrollo ha sido validado en la ampliación de las perspectivas del consultante y en los hallazgos de las similitudes entre los personajes y su propia historia, incitándolo de esta forma a la expresión de sus sentimientos. La biblioterapia, en términos disciplinares, mantiene entre sus objetivos la mejora terapéutica a través del uso del texto. Es la lectura - y la posibilidad ilimitada que conlleva - una actividad de fines propositivos y una respuesta frente a angustias de corte existencial (Román, 2019; Román, 2020).

La construcción del cambio y el desarrollo de nuevas acciones de comportamiento que amplían el mapa mental postulan un desarrollo y una mirada más abarcativa de su problemática y del contexto a través del libro. Frankl sostiene que la 
actividad de la lectura queda completamente asociada a la búsqueda de sentido y, por eso, puede desprenderse de ella que posee un gran valor terapéutico. En sintonía, la dimensión del libro otorgada por Clemente (1964) favorece y permite vivencias que se proyectan sobre el lector, aportando mundos diferentes y sensación de libertad.

Tanto en la mirada sobre relaciones interpersonales como sobre relaciones de pareja, los terapeutas se valen de textos específicos que favorezcan la disminución del conflicto y la construcción de vínculos saludables y asertivos.

La elaboración de duelos goza de una significación especial en la recomendación terapéutica al incluir sugerencias de textos empáticos que orientan al permiso del atravesamiento de las diferentes etapas del proceso de pérdida. La comprensión de los sentimientos en este sentido tranquiliza al paciente y le permite recorrer estas instancias en términos de procesos que incluyan la autocomprensión. Los aportes de Bardají (2016) sobre biblioterapia y duelo postulan de modo estratégico una percepción más amplia del vivir y del enfoque de la comprensión del ser humano y su inclusión en un universo amplio. E incluso los desarrollos de Kroeff y Reverté (2007) acerca del dispositivo en el cual examinan ciertas frases tomadas de los libros amplian los recursos a través del espacio para abordar circunstancias de duelo en grupos psicoterapéuticos accedidos por personas con viudez reciente.

Ya sea desde el aumento de los recursos con los que el cliente cuenta como desde la modificación de creencias rígidas sin evidencia, como en la toma de conciencia de disfunciones y distorsiones, la biblioterapia ofrece un cambio ameno y amigable en el que la lectura muestra un es posible.

Los aportes de Román (2019) que asocian las representaciones de exreclusos sobre la lectura y el sentido de la vida exhiben, a su vez, otros episodios colegidos en su investigación respecto de esta línea de trabajo. Asimismo, Del Río (2011) aporta un dispositivo de lectura destinado a grupos de tercera edad, que fomenta el contacto con el sentido de la vida. Producciones como las de Román y Del Río dan cuenta de las múltiples y apreciables usanzas de la biblioterapia.

Tanto en el abordaje de neurosis noógenas como en tratamientos psicoterapéuticos de abordaje de diferentes patologías, en modalidades individuales o grupales, en 
dispositivos de consultorios $\mathrm{u}$ hospitalarios, las recomendaciones de libros constituyen acervos que empoderan al paciente, ya sean lecturas en papel o digitales. Investigaciones como las de Deberti Martins (2011) dan cuenta de su uso en espacios de organizaciones de salud y en el ámbito hospitalario.

El enfoque de empleo de la biblioterapia como autoterapia ampliada coincide con Losada (2021) en continuar el trabajo iniciado en el espacio terapéutico, prolongando de alguna forma la sesión y los temas trabajados de acuerdo con las motivaciones del grupo participante.

Congruentemente, las recomendaciones de libros en la autoterapia ampliada pueden extenderse a los espacios grupales y constituirse como herramienta válida en los grupos psicoeducativos. En estos grupos se fijan objetivos y los programas ofrecen sugerencias de textos preestablecidos y de sesiones semanales $\mathrm{o}$ quincenales (Losada y Zavalia, 2019).

\section{Consideraciones finales}

Atento a que la sociedad en su totalidad debiera comprender que todos sus integrantes tienen el derecho a las mismas posibilidades, respetando sentimientos, valores y principios (Losada y Rijavec, 2017), es la lectura un recurso de acceso factible y comunitario, cuyo único requisito es haber alcanzado la alfabetización. El intento por reunir las nociones de varias técnicas para abordar mejor un problema es lo que se debe buscar a la hora de asistir al paciente. Estar abierto a la contingencia de emplear lo mejor de cada enfoque es de vital importancia para la eficacia del proceso curativo (Losada, 2013).

Destaca Deberti Martins (2013) el fenómeno de la lectura en general y, particularmente, lo inherente a su empleo reparador del tejido social y del psiquismo a través del proceso de simbolización. Es en este sentido de contribuir a la mejora y bienestar social a través de la psicología que se postula el uso de la recomendación terapéutica y el libro como una herramienta altamente eficaz, capaz de potenciar el trabajo de las sesiones entre un encuentro y el próximo. 


\section{Referencias bibliográficas}

Bardají, A. F. (2016). Cómo afrontar la pérdida con Proust: un apunte de biblioterapia. Escritos de Psicología, 9(3), 60-67.

Clemente, J. E. (1964). Estética del lector. Buenos Aires: El Ateneo.

Deberti Martins, C. (2011). Leer: un derecho... también en el hospital. Información, Cultura y Sociedad, (25), 145-152.

Deberti Martins, C. (2013). ¡Los libros muerden! Biblioterapia en el portal amarillo. Informatio. Revista del Instituto de Información de la Facultad de Información y Comunicación, 18(1), 21-30.

Del Río, G. M. (2011). El valor de la biblioterapia en la vejez. Revista Portal De Divulgação, (15), 25-33.

Frankl, V. (2012). Psicoterapia y humanismo. Ciudad de México: Fondo de Cultura Económica.

Frank1, V. (2013). El hombre en busca de sentido. Barcelona: Herder.

Kroeff, P., y Reverté, M. A. L. (2007). Biblioterapia con personas mayores en grupo pos-duelo. Boletín de Logoterapia y Análisis Existencial, 51-67.

Losada, A. V. (2014). Uso en investigación y psicoterapia del consentimiento informado. En B. Kerman, y M. R. Ceberio (Comps.), En búsqueda de las ciencias de la mente: investigación en psicología sistémica, cognitiva y neurocientífica (pp. 159-167). Buenos Aires: Ediciones Universidad de Flores.

Losada, A. V. (2020). La biblioterapia como recurso terapéutico interdisciplinario. Revista de Psicología, 19(1), 210-221.

Losada, A. V. (2021). Distorsiones cognitivas alimentarias. En H. Fernández Álvarez (Ed.), Enciclopedia de salud mental. Buenos Aires: Aiglé.

Losada, A. V., y Rijavec, M. I. (2017). Consecuencias psicológicas en niños con obesidad producto de la estigmatización social. Revista Neuronum, 3(2), 46-65.

Losada, A. V., y Saboya D. (2013). Abuso sexual infantil, trastornos de la conducta alimentaria y su tratamiento. Psicología, Conocimiento y Sociedad, 3(2), 102-134. 
Losada, A. V., y Zavalia, C. (2019). Programa psicoeducativo en trastornos de la conducta alimentaria. Revista Alternativas en Psicología, (41), 131-146.

Román, A. M. (2019). Imaginarios sociales de exreclusos sobre la lectura y el sentido de la vida: la función de la biblioterapia en personas privadas de su libertad (Tesis doctoral). Universidad Argentina John F. Kennedy, Buenos Aires.

Román, A. M. (2020). Revisión sistemática de artículos sobre biblioterapia y el sentido de la vida. Calidad de Vida y Salud, (13) 114-129.

\section{Notas del editor}

El presente manuscrito fue aprobado para su publicación por Cristina Deberti.

La corrección de estilo del presente texto fue realizada por Elena Ponte en el marco del convenio celebrado entre la FHCE (Tecnicatura Universitaria en Corrección de Estilo-Facultad de Humanidades y Ciencias de la Educación) y la FIC (Facultad de Información y Comunicación).

\section{Nota de contribución autoral}

Las autoras declaran haber participado igualitariamente en la elaboración del presente manuscrito. 